\title{
MicroED for small molecule and natural product research
}

\author{
E Danelius ${ }^{1}$, J Hattne ${ }^{2}$, M Martynowycz ${ }^{3}$, S Halaby ${ }^{4}$, T Gonen ${ }^{5}$ \\ ${ }^{1}$ UCLA, Los Angeles, CA, ${ }^{2}$ University of California, Los Angeles, ${ }^{3}$ HHMI/UCLA, Los Angeles, CA, \\ ${ }^{4}$ UCLA Biological Chemistry, Los Angeles, CA, ${ }^{5} \mathrm{HHMI} / \mathrm{UCLA}$ \\ danelius@ucla.edu
}

The electron cryo-microscopy (cryo-EM) method Microcrystal Electron Diffraction (MicroED) allows the collection of high-resolution structural data from vanishingly small crystals, typically with a thickness in the nanometer range. Since its debut in 2013, data collection and analysis schemes have been fine-tuned, and there are now over 100 structures determined by MicroED. Although originally developed to study proteins, MicroED is clearly also powerful for smaller systems, with some recent and very promising examples of important natural products and peptides as well as small molecule pharmaceuticals. As for any structural investigation by X-ray crystallography, deriving the structures of small molecules and natural products is often prohibited due to difficulties in obtaining the large and well-ordered crystals required. On the other hand, the crystals used for MicroED are about a billionth the size as compared to those used for X-ray diffraction which means that they are much more easily obtained, as demonstrated by the many novel and previously unattainable structures. In fact, in MicroED atomic resolution structures can rapidly be derived directly from powders, i.e. without any prior crystallization. In these cases, seemingly amorphous solids from silica gel chromatography or directly from a chemical supplier were used. In this seminar I will show recent examples of MicroED in small molecule research including determining the stereochemistry of natural products, as well as deriving the atomic structures of molecules directly from compounds mixtures, where the latter example is not possible with other structural methods. Given the unique features of MicroED, its potential impact is multifold, and the method is expected to continue to greatly influence the field of natural product and small molecule research.

Acta Cryst. (2020). A76, a185 